\title{
VLT/NACO detection of a proplyd/jet candidate in the core of Trumpler 14
}

\author{
Sílvia Vicente ${ }^{1}$, João Alves ${ }^{2}$, Isamu Matsuyama ${ }^{3}$, \\ Hervé Bouy $^{4}$, Loredana Spezzi ${ }^{1}$, Joana Ascenso ${ }^{5}$, Filipe D. Santos ${ }^{6}$ \\ and Timo Prusti ${ }^{1}$ \\ ${ }^{1}$ ESA (ESTEC), Research and Scientific Support Department, \\ Keplerlaan 1, P.O. Box 299, 2200 AG Noordwijk, The Netherlands \\ email: svicente@rssd.esa.int \\ ${ }^{2}$ Institut für Astronomie, Universität Wien, \\ Dr.-Karl-Lueger-Ring 1, 1010 Wien, Austria \\ ${ }^{3}$ Department of Earth and Planetary Sciences, University of California Berkeley, \\ 307 McCone Hall, Berkeley, CA 94720, USA \\ ${ }^{4} \mathrm{CAB}$ (INTA/CSIC), LAEFF, \\ P.O. Box 78, E-28691 Villanueva de la Cañada, Madrid, Spain \\ ${ }^{5}$ European Southern Observatory, \\ Karl-Schwarzschild Straße 2, D-85748 Garching bei München, Germany \\ ${ }^{6}$ Departamento de Física da Faculdade de Ciências da Universidade de Lisboa, \\ Ed. C8, Campo Grande, 1749-016 Lisboa, Portugal
}

\begin{abstract}
This paper reports the discovery and presents the results of a first analysis of the observed morphology of a candidate external irradiated circumstellar disk/jet system found in the deep core of Trumpler 14, a cluster an order of magnitude more massive than the only cluster where bona-fide proplyds have been found, the Trapezium cluster in the Orion Nebula.
\end{abstract}

Keywords. HII regions, planetary systems: protoplanetary disks, ISM: globules, ISM: jets and outflows, stars: formation, open clusters and associations: individual (Trumpler 14)

The proplyd/jet candidate was discovered during a VLT/NACO $J H K s L^{\prime} \mathrm{Br} \alpha$ survey of the core of Trumpler 14 (Vicente et al. 2010, Ascenso et al. 2007), a young cluster in the Carina Nebula (NGC 3372), and is similar in size and morphology to the numerous proplyds found in the Trapezium cluster (Bally et al. 2000, Vicente \& Alves 2005). Archival HST/ACS/HRC optical images, together with the adaptive optics near-IR images and existing photoevaporation theories, were used to investigate the morphology of the object and the possible scenarios for its nature, origin and expected lifetime.

The evaporating globule is located very close to the O2If* supergiant star, HD 93 129Aa, at a projected distance of $\sim 0.024 \mathrm{pc}$. Visible as a tailless object in the optical emission lines, [OIII] and $\mathrm{H} \alpha$, as a faint point source in $H$ and with a faint tail in $K_{s}$, it appears in the $L^{\prime}$ band and $4.05 \mu \mathrm{m}$ images as an extended object with a bright "head" and a long irregular, clumpy tail pointing nearly radially outwards from HD 93129A (Fig. 1). In the $L^{\prime}$-band image, the object's head has a diameter of $560 \mathrm{AU}$ and a head-to-tail length of $3080 \mathrm{AU}$, for the adopted distance of $2.8 \mathrm{kpc}$ to Trumpler 14. A spike emerging from the head and a 2-tail morphology, also visible in the deconvolved image, are suggestive of the presence of a jet. Are the two sections of the tail associated with different physical processes? Possible scenarios for the observed morphology include: 1) a jet + proplyd evaporative flow, 2) a precessing jet/disk, and 3) a non-resolved binary driving two jets. With the present data we cannot state beyond any doubt that this object is a true 

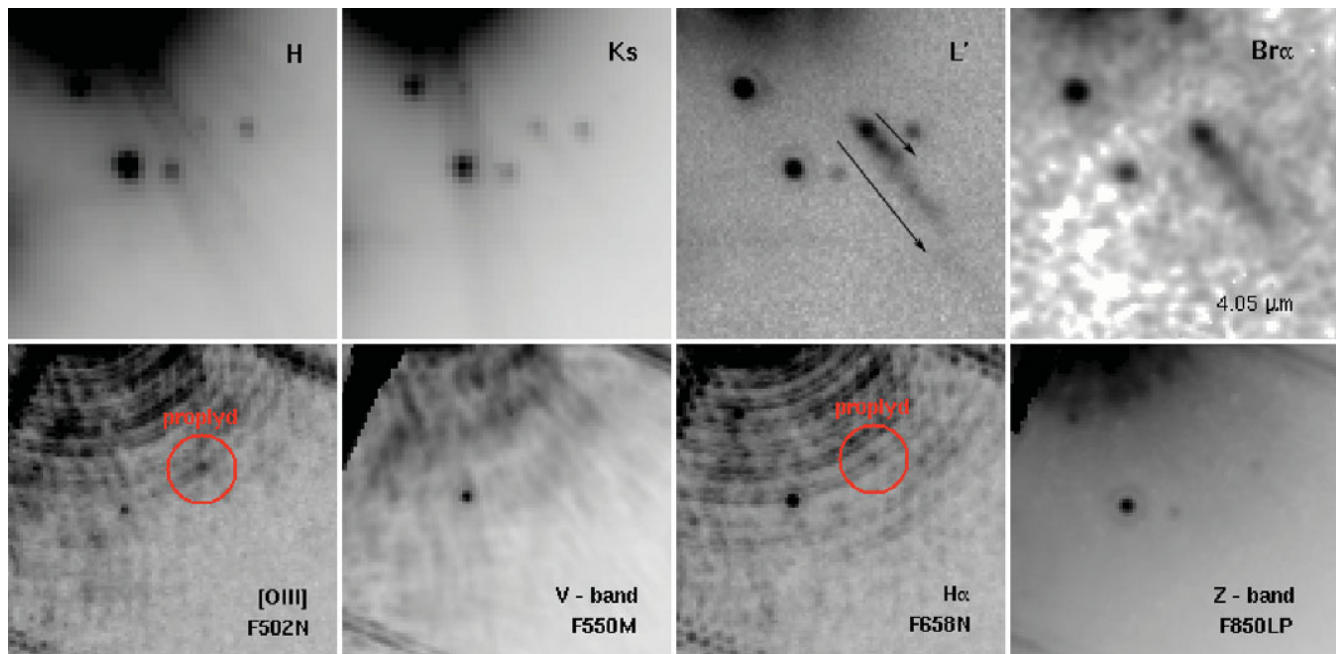

Figure 1. VLT/NACO near-IR images (upper row, $2 " .5 \times 2 " .5$ ) and HST/ACS/HRC optical images (bottom row, 2 ". $3 \times 2$ ". 3 ) of the proplyd/jet candidate in the core of Trumpler 14. North is up and east to the left. A spike emerging from the globule's head and a 2-tail morphology in the $L^{\prime}$-band image are suggestive of the presence of an externally illuminated bipolar jet where dust is entrained. Tail1 is shorter and brighter with a head-to-tail extent of 0.5 " (1400 AU), and tail2 is longer (1.1" or $3080 \mathrm{AU}$ ), fainter, and nearly parallel to the former. Their length and direction are indicated by the black arrows in the $L^{\prime}$-band image.

proplyd/jet as opposed to an evaporating gaseous globule but photoevaporation massloss rates, predicted at that location, favor a very compact and dense system, and hence, the proposition that it is indeed a proplyd. A 560 AU spherical globule of molecular hydrogen would be disrupted and completely evaporated in less than 50 yr. Future detailed high-resolution multi-wavelength observations are required to determine accurately the physical parameters of this intriguing object and will lead to a better understanding of its nature.

Other proplyd candidates have been discovered in the core of Trumpler 14 in HST/ACS images (S. Vicente PhD thesis 2009, Smith et al. 2010). This discovery is surprising since Trumpler 14 is older (1-2 Myr, Smith et al. 2006) than the Trapezium (0.1-1 Myr), a much more extreme environment $\left(Q_{H} \sim 22\right.$ times larger $)$ and has a PDR located at $2 \mathrm{pc}$ from the cluster core (Brooks et al. 2003). The survival of these candidate protoplanetary disks raises many questions related to the current photoevaporation theories developed for the Trapezium proplyds. Can we apply the same models to the harsh environment of Trumpler 14? What is missing in the whole picture?

\section{References}

Ascenso, J., Alves, J., Vicente, S., \& Lago, M. T. V. T. 2007, A\&A, 476, 199

Bally, J., O'Dell, C. R., \& McCaughrean, M. J. 2000, AJ, 119, 2919

Brooks, K. J., Cox, P., Schneider, N., et al. 2003, A\&AA, 412, 751

Smith, N. 2006, MNRAS, 367, 763

Smith, N., Bally, J., \& Walborn, N. R. 2010, MNRAS, 405, 1153

Vicente, S. \& Alves, J. 2005, A\&SA, 441, 195

Vicente, S. 2009, PhD Thesis, Faculty of Sciences of Lisbon University

Vicente, S., Alves, J., Matsuyama, et al. 2010, submitted to A\&A 